RHIC-PG-9

RHIC MAGNET DESIGN STUDY

H. HAHN

(BNL, November 23, 1983) 
H. Hahn

Nov. 22, 1983

\section{RHIC MAGNET DESIGN STUDY}

OBJECTIVES: $\quad$ Reduce Cost

Establish Dependence of cost on aperture, field and unit length (discontinuities?)

Dipoles and quadrupoles

Decision in March ( $\& \& D$ and construction cost)

New Magnet 12M\$ and 18 months delay (?)

2-in-1 CBA 6M\$ and 6 months delay (?)

METHOD :

Fill tunnel, Iower field

Reduce aperture, bunched beams

Use other concepts

FNAL magnet

window frame (super ferric),

SSC

Conventional magnets

Different construction (BBC, HERA magnet)

Single layer CBA quadrupole

FUTURE MEETINGS: R. P. Shutt 
H. Hahn

Nov. 22, 1983
TENTATIVE GUIDELINES FOR RHIC MAGNET

- 2-in-1 physical configuration

- Separate magnetic excitation; operate at $2.5: 1$ ratio

- Horizontal beam crossing; spacing

- Magnet Length (CBA)

- Field@ $100 \mathrm{GeV}$

(higher if lumped trim coils or

more quadrupoles for higher transition energy)

- Field range

- Coil id. in arc

Beam size @ $5 \mathrm{GeV}$ on $6 \mathrm{~mm}$

"7 $\sigma "$ rule, sagitta, momentum spread

Intra-beam scattering

- Operating temperature

$4.6 \mathrm{~K}$

- Probably cold bore in arc (insertions?)

- Straight magnets

(gain in aperture if curved)

- Field quality

$1 \times 10^{-4} \mathrm{rms}$ at $2 / 3$ aperture and low field

$10^{-3} \mathrm{rms}$ at $1 / 3$ aperture and high field

- Trim Coils (lumped or distributed?)

dipole correctors

quadrupole - separate bus for horizontal and vertical quads

sextupole

$b_{o}, b_{2}:$ if distributed - single layer enough

$\left.\begin{array}{l}\text { Skew Quad } \\ \text { Octupole }\end{array}\right\}$

- Q1, Q2 non standard aperture

approximately present size
$24 \mathrm{~cm}$

$4.4 \mathrm{~m}$ effective

$33 \mathrm{kG}$

$20: 1$

3 in. 